\title{
The Trends of Viral Hepatitis B and C and HIV Infections in Donated Bloods in Iran Between 2003 and 2017
}

This article was published in the following Dove Press journal:

Journal of Blood Medicine

Moslem Taheri Soodejani (D)

Ali Akbar Haghdoost'

Abbas Sedaghat ${ }^{2}$

Mohammad Reza Baneshi $\mathbb{D}^{3}$

Farzaneh Zolala ${ }^{4}$

'HIV/STI Surveillance Research Center, WHO Collaborating Center for HIV

Surveillance, Institute for Futures Studies in Health, Kerman University of Medical Sciences, Kerman, Iran; ${ }^{2}$ Blood

Transfusion Research Center, High Institute for Research and Education in

Transfusion Medicine, Tehran, Iran;

${ }^{3}$ Modeling in Health Research Center,

Institute for Futures Studies in Health,

Kerman University of Medical Sciences, Kerman, Iran; ${ }^{4}$ Social Determinants of

Health Research Center, Institute for

Futures Studies in Health, Kerman

University of Medical Sciences, Kerman, Iran
Correspondence: Farzaneh Zolala Institute for Futures Studies in Health, Kerman University of Medical Sciences, Kerman 7616913555, Iran

Tel +983431325700

$\mathrm{Fax}+983431325135$

Email zolalafarzaneh@gmail.com
Background: The rate of viral infections in donated bloods is one of the main indicators of blood safety which has to be monitored precisely. This paper provides a thorough study of blood safety indicators in the last 15 years in Iran.

Methods: The data of the transfusion organization in Iran from years 2003 to 2017 were used. The study focuses on the analysis of the frequency of viral hepatitis B and C (HBV and $\mathrm{HCV}$ ) as well as human immunodeficiency virus (HIV) in blood donations.

Results: Of 27,442,124 donated bloods, the relative frequencies were 4, 302, and 81 for $\mathrm{HIV}, \mathrm{HBV}$ and HCV, respectively. This study also shows that the corresponding frequencies were significantly lower in recent years $(2.5,53$, and 26 per 100,000 samples in 2017).

Conclusion: The presented study indicates an overall low infection rate and provides evidence for the effectiveness of modern safety measures in improving the level of blood safety in Iran.

Keywords: transfusion-transmitted infections, infection, blood donors

\section{Introduction}

Nearly $30 \%$ of humans at some period of their lifetime require the use of blood or blood products in order to continue living. Despite the necessity of blood products in certain medication circumstances, unhealthy blood products can cause serious risks for the patients or even endanger their lives. ${ }^{1}$

Blood presents a potential risk for the receivers of transfusions. The rate of viral infections in donated bloods plays a key role as the main indicator of blood safety, which has to be monitored precisely. The biggest challenge in this regard is transfusiontransmitted infections (TTIs), the most important of which are human immunodeficiency virus (HIV) as well as hepatitis types $\mathrm{B}(\mathrm{HBV})$ and $\mathrm{C}(\mathrm{HCV})$ viruses which create a large burden for the healthcare system. ${ }^{2,3}$ Based on the WHO categorization, Iran which is one of the countries in the EMRO region has a population of more than 80 million people. Iran is prone to natural disasters and some of them lead to considerable injuries. ${ }^{4}$ Moreover, car accidents are one of the leading causes of death among the Iranian. ${ }^{5}$ Therefore, the Iran Blood Transfusion Organization (IBTO) has a crucial role in providing healthy blood to those in need.

According to the literature, the estimated prevalence of $\mathrm{HBV}$ and $\mathrm{HCV}$ is 2.2 and $1.6 \%$ among Iranian general population, respectively. This prevalence in the general population can affect the prevalence in blood donors. ${ }^{6,7}$ 
Given the significance of these infections and the ease with which they are transmitted through blood products, the IBTO follows a set of standard operation procedures (SOPs) to identify infected blood donations and prevent their use. These procedures include two main activities: selecting safe donors and performing screening tests in order to identify TTIs. ${ }^{8}$

The selection process includes interviewing each volunteer by a qualified interviewer. In case of any risk factors or any history of risky behaviors, blood donation will be refused. ${ }^{9,10}$ In the next step, donated bloods are going to be screened for TTIs.

These tests investigate screening the HCV Ab, HBsAg and HIV $(\mathrm{Ag} / \mathrm{Ab})$ using the ELISA test according to the IBTO guidelines. ${ }^{3,10}$

The screening method used in IBTO to screen donated blood has been improved over time base on new technologies of screening methods. For example, prior to 2010, screening tests were carried out using portable ELISA testing kits which lacked the necessary accuracy due to human error. However, between the years 2009 and 2010, the method shifted from the use of manual testing kits to the use of the automation method. This intervention created a new screening procedure which removed the human error and increased the accuracy in reporting confirmed TTIs result tests, and hence, led to much more accurate and reliable test results.

In order to assess the effects of upgrading the mentioned screening methods, it is needed to develop a plan to analyze the situation of screening results, before and after this switching. The effectiveness of switching the methods of the TTIs screening system has been analyzed by the interrupted time series (ITS) model.

This study was conducted to show the effect of the new screening method on identifying new cases of TTIs. This is the first report of the rate of TTIs in the national level among Iranian donated bloods.

\section{Methods}

\section{Study Design and Data Collection}

This study used the data registered in the donor vigilance part of IBTO. The data include the number of donors and the number of identified infections between the years 2003 and 2017 for each province. The data were accessed through coordination with IBTO.

The data from each province were sent to the Iranian blood transfusion center monthly, which has a $100 \%$ coverage and covers all donors in Iran. It should be mentioned that the tests used to identify TTI have a high sensitivity and specificity and also identify almost all people with these infections.

\section{Data Analysis}

The improvement in the identification of TTIs resulting from changes in screening procedures was investigated using the interrupted time series (ITS) model. The model included four parameters which are explained in the next section. The results for three main infections (HIV, HCV and HBV) are then visualized using bar charts created by MS Excel.

In this model (see Formula 1), the $\beta_{0}$ parameter is the intercept which indicates the level of identified infections at zero point (the year 2003). Furthermore, $\beta_{1}$ shows the pre-intervention trend which is the trend in the identification of TTIs before the introduction of new screening procedures. This parameter shows the changes in the prevalence of TTIs each year before the intervention. The $\beta_{2}$ parameter shows the change in the intercept immediately after the intervention (new screening procedure) which indicates the changes in the TTIs prevalence immediately after the use of new screening procedures. Furthermore, $\beta_{3}$ is the change in the post-intervention trend which indicates the changes in the TTIs prevalence after the use of new screening procedures compared to expected changes without intervention. This parameter shows the changes in the prevalence of each TTI each year compared to the preintervention trend (the previous screening procedures). Finally, $\varepsilon$ is the residual error of the model. ${ }^{11}$

$$
\text { outcome }=\beta_{0}+\beta_{1}(\text { time })+\beta_{2}(\text { level })+\beta_{3}(\text { trend })+\varepsilon
$$

Furthermore, a color spectrum based on quarters was used to determine the geographical distribution of TTIs in donors of each of the 31 provinces of Iran. The first quarter (Q1) indicates the lowest prevalence while the fourth quarter (Q4) has the highest prevalence in the provinces. This chart was created using the Arc Map GIS Ver. 10.2 software.

\section{Results}

\section{Descriptive Analysis}

Between the years 2003 and 2017, a total of 27,442,124 blood donations (overall, about $20 \%$ of donors are firsttime donors, and the remaining $80 \%$ have had blood donations more than once before, this ratio that has been almost constant in recent years) were carried out in Iran. 
Of this number, a total of 1063 cases of HIV, 82,989 cases of HBV and 22,275 cases of HCV were identified in the donated blood samples (see Figure 1).

The period prevalence (15 years) of these infections was 4, 302 and 81 cases per 100,000 donations for HIV, $\mathrm{HBV}$ and $\mathrm{HCV}$, respectively. The largest number of infections was identified in the initial years of the study (2003 to 2006) while the number of infections was lowest in the year 2017 (2.5, 53 and 26 cases in 100,000 donations for HIV, HBV and HCV, respectively). Among these infections, HBV had the highest prevalence (Figure 1).

Investigating the geographical distribution of TTIs in Iranian blood donors showed that all the three infections had the highest prevalence near Iran's western and eastern borders (see Figure 2).

\section{Intervention (New Screening Procedure)}

Investigating the prevalence trends before and after the intervention showed an interaction between time period and intervention which was statistically significant $(\mathrm{P}<0.01)$. Since the interaction was significant, two slopes were needed to examine the trend. Therefore, the ITS approach was used to investigate the prevalence trends before the intervention (the new screening method) and compare the results before and after the intervention.

The prevalence of TTIs before the intervention (the new screening method) showed a decreasing trend between the years 2003 to 2009 . At the time of the intervention, the prevalence for $\mathrm{HCV}, \mathrm{HBV}$ and $\mathrm{HIV}$ were
$173.1,895$ and 6.3 cases per 100,000 donations. This preintervention trend was shown in the trend graph as a dotted line and decreased at a constant rate. After the introduction of the intervention (the new screening method) between the years 2009 and 2010, this trend was interrupted and replaced by a new trend (the line in the graph separating the years 2009 and 2010). This new trend showed a more positive change in prevalence immediately after the intervention for all the infections except $\mathrm{HCV}$.

After the introduction of the intervention (the new screening method), the new trend for infection prevalence was still decreasing but showed a constant change compared to the pre-intervention trend. This constant changed compared to the previous year's results in the identification of $61.4,4.5$ and 0.25 more cases of HBV, HCV and HIV compared to the expected pre-intervention values which are statistically significant $(\mathrm{P}<0.001)$.

Figure 3 shows the changes in the prevalence trends before and after the intervention while the parameters for each infection are presented in Table 1 .

\section{Discussion}

In order to better understand the interpretations, the discussion is divided into two parts. The first part investigates the prevalence of TTIs while the second part investigates the effect of the intervention in the identification of TTIs using the ITS model.

The results indicated that the prevalence of HBV (302 cases per 100,000 donations) was higher than that of HCV

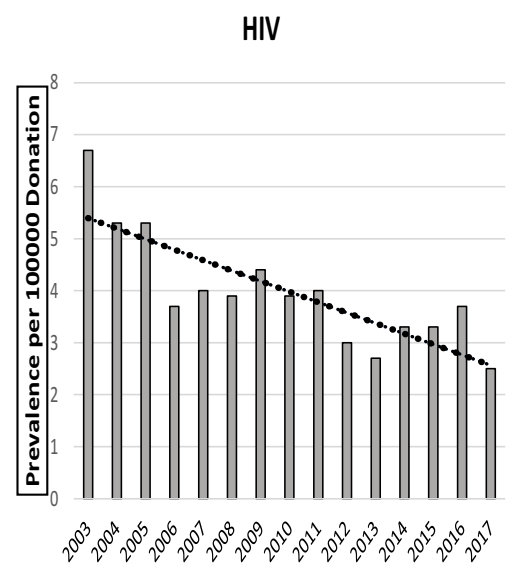

Year

Standardized Beta $=-0.82$

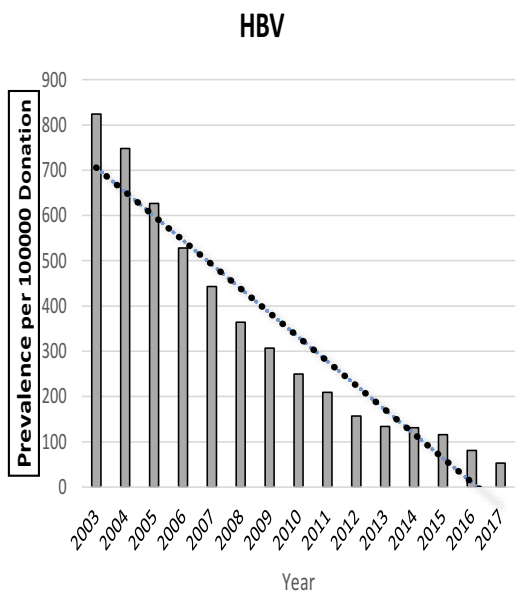

Standardized Beta $=-0.96$

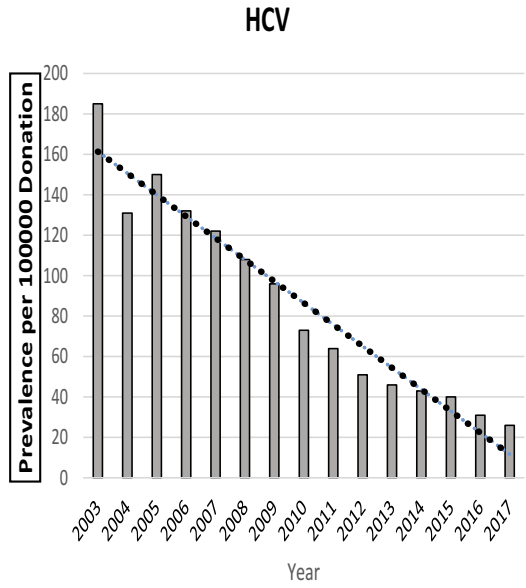

Standardized Beta $=-0.97$

Figure I The trend of prevalence of TTIs in Iranian donated bloods from 2003 to 2017

Note: The Y-axis scale is different in each graph. 


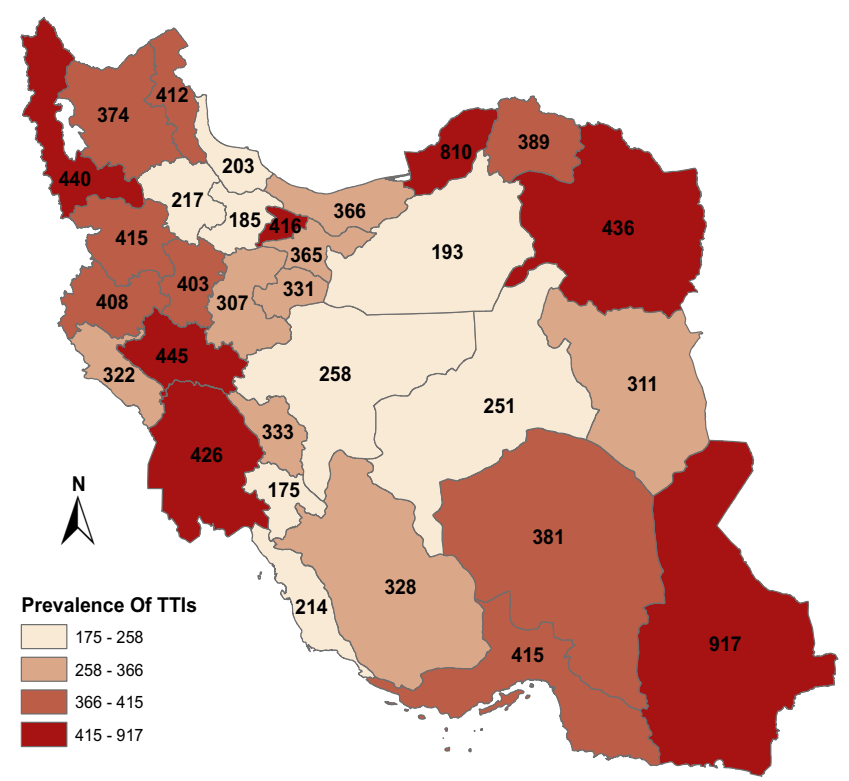

Figure 2 The geographic distribution of the period prevalence (2003-2017) of TTls in Iranian donated bloods (per 100,000 donations).

(81 cases per 100,000 donations) in Iranian donated bloods in the study period (15 years) while the prevalence of HIV (9 cases per 100,000 donations) was lower than that in blood donors in most other countries. Other studies in India $(\mathrm{HBV}=1.77 \%, \mathrm{HIV}=0.14 \%$ and $\mathrm{HCV}=0.04 \%)$, Colombia $(\mathrm{HBV}=0.12 \%, \mathrm{HCV}=0.45 \%$ and $\mathrm{HIV}=0.12 \%)$, Italy $(\mathrm{HBV}=0.01 \%)$ and Turkey $(\mathrm{HBV}=4.19 \%$ and $\mathrm{HCV}=0.38 \%)$ showed a higher prevalence of TTIs among blood donors compared to Iranian donors. ${ }^{12-15}$

Blood donors can be considered as a representative of the general population of the country. Even though the demographic characteristics of blood donors can differ from those of the general population, these factors have a slight effect on the distribution of TTIs. One of the reasons for the relatively low prevalence of TTIs among Iranian donors might be the low prevalence of these infections among Iran's general population compared to other countries. For example, systematic review studies on the prevalence of $\mathrm{HBV}$ among the WHO member countries showed that the prevalence of HBV in India (1.4\%), Italy (2.52\%), Colombia (2.3\%) and Turkey (4\%) is significantly higher than the HBV prevalence in Iran $(0.96 \%) .{ }^{16}$

Another reason for the difference in the prevalence rate can be the difference in the blood donor population. Blood donation in Iran is fully voluntarily and donors receive no payments. Therefore, blood donors are often people with better health situations who participate without expecting financial compensation. However, in other countries, blood donation is sometimes due to monetary incentives. For example, a study in India showed that only $8 \%$ of blood donations were voluntary. ${ }^{14}$ Usually, people who participate in blood donation due to financial incentives have lower health conditions, which can explain the higher prevalence of TTIs among these people.

The prevalence of TTIs among Iranian blood donors showed a decreasing trend between the years 2003 and 2017. The results of other studies on the prevalence trends of TTIs among Iranian blood donors are similar to the results of our study and show a decreasing trend of TTIs among Iranian blood donors. ${ }^{1,17}$

Although the results of the current study did not distinguish between different types of donors in the initial years (2003 to 2010), other studies conducted in some of the Iranian provinces in the years 2005 and 2006 showed that the highest prevalence of TTIs was observed in the

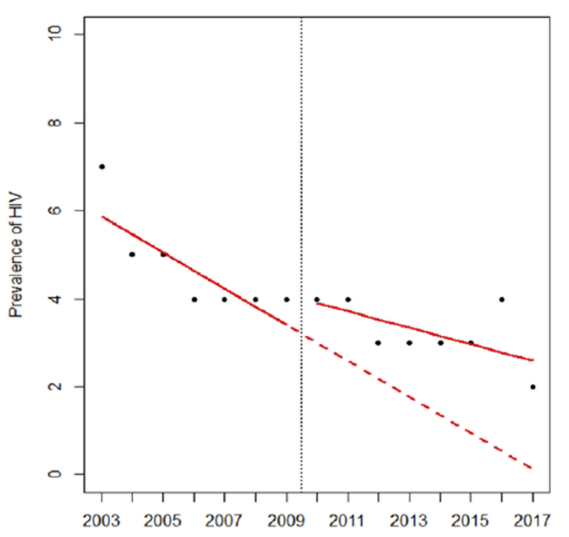

A

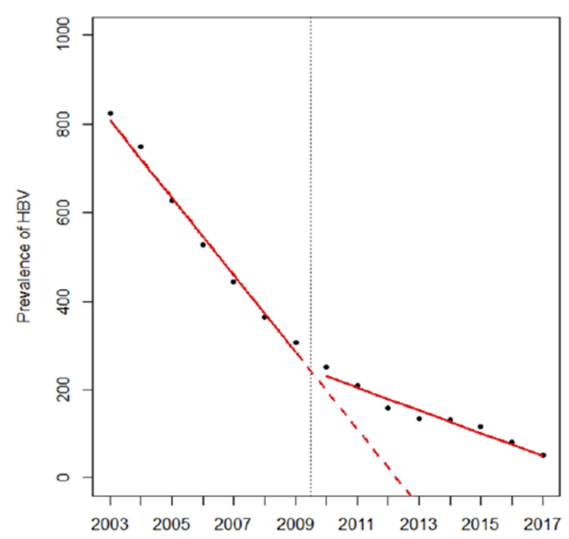

B

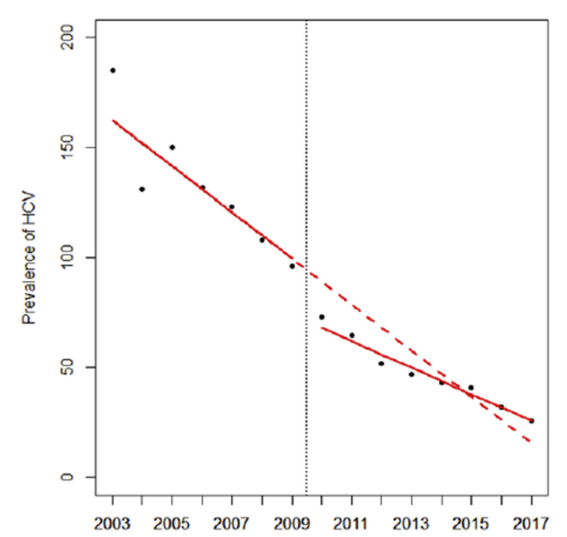

Cear

Figure 3 The interrupted time series analysis of HIV (A), HBV (B), HCV (C) in Iranian donated bloods. 
Table I ITS Parameters in the Trend of TTIs in Iranian Donated Bloods

\begin{tabular}{|c|c|c|c|c|}
\hline \multirow[t]{5}{*}{ HIV } & Coefficient & Value & Standard Error & $\mathbf{P}$ \\
\hline & Intercept & 6.3 & 0.4 & $<0.001$ \\
\hline & Pre-intervention trend & -0.4 & 0.1 & $<0.001$ \\
\hline & Change in intercept immediately after intervention & 0.52 & 0.5 & 0.3 \\
\hline & Change in the post-intervention trend & 0.25 & 0.1 & 0.02 \\
\hline \multirow[t]{5}{*}{ HBV } & Coefficient & Value & Standard Error & $\mathbf{P}$ \\
\hline & Intercept & 895 & 7.4 & $<0.001$ \\
\hline & Pre-intervention trend & -87.2 & 1.7 & $<0.001$ \\
\hline & Change in intercept immediately after intervention & -28.3 & 9.2 & 0.01 \\
\hline & Change in the post-intervention trend & 61.4 & 1.9 & $<0.001$ \\
\hline \multirow[t]{5}{*}{$\mathrm{HCV}$} & Coefficient & Value & Standard Error & $\mathbf{P}$ \\
\hline & Intercept & I73.I & 2.8 & $<0.001$ \\
\hline & Pre-intervention trend & -10.5 & 0.6 & $<0.001$ \\
\hline & Change in intercept immediately after intervention & -25.5 & 3.4 & $<0.001$ \\
\hline & Change in the post-intervention trend & 4.5 & 0.7 & $<0.001$ \\
\hline
\end{tabular}

first-time donors. ${ }^{18}$ Another study in Shiraz (in the southwest of Iran) in 2004 showed that nearly $15 \%$ of blood donors participated in a donation in order to test for HIV. ${ }^{19}$

It appears that as years go by, a larger ratio of donors is made up from repeat and regular blood donors. These people receive more education compared to first-time blood donors, which can explain a lower prevalence of TTIs.

The geographical distribution of the period prevalence ( 15 years) of TTIs among Iranian donors showed that locations closest to Iran's western and eastern borders had a higher prevalence of the infections. Various studies in Iran and in different provinces also showed a higher prevalence of the infections near the western and eastern borders. ${ }^{6,7,20,21}$

The second part of the discussion focuses on the effects of the intervention (the new screening method) and the interpretation of the ITS results.

The screening activities for blood donors have improved over time in order to ensure access to healthy blood products which can save the lives of patients. The main change in the screening pattern for TTIs in blood donations in Iran occurred between the years 2009 and 2010, during which testing methods changed from the use of manual portable testing kits to the use of automated approach which improved the precision and accuracy of the tests several folds.

Investigating the prevalence trends of TTIs at different time periods (before and after the intervention) showed that there was an interaction between time and intervention which revealed two different trends in these different periods. Therefore, the ITS approach was used to investigate the effect of the new screening method.

Using ITS mode, we found out that the number of the identified cases for HBV and HIV increased immediately after the intervention. We hypothesized that it was more likely to have an improvement in the screening method rather than increasing the new cases. The reason is that the coefficient of "change in the intercept immediately after the intervention" showed an immediate change after the intervention and not an ongoing change.

However, this effect was different in the case of HCV and only showed an increase in the number of the identified cases some years after the implementation.

The relatively high prevalence of HBV compared to the other two infections means that any changes in its prevalence trends over the years were more visible and that these changes could be interpreted more readily. If the prevalence trend observed for HBV before the intervention (the new screening method) was required to continue, it was expected to detect no new cases of HBV by the year 2013. However, the total detected cases of HBV in the year 2013 were 134 cases in every 100,000 blood donations. The difference between the expected values (in the absence of the intervention) and the actual number of the identified cases showed the effect of the new screening method.

Although the prevalence of HCV and HIV was relatively low compared to HBV, it was also expected for the 
new detected cases of these infections to be zero without the use of the new screening method. Blood donors turn into repeat and regular donors after some years. Such people are usually those who avoid risky behaviors and live healthier lives compared to other members of the society. $^{22,23}$ Furthermore, some programs introduced by the IBTO focus on educating and improving the awareness of blood donors. ${ }^{24}$ As a result, the risk of being afflicted with TTIs is lower in these people. All of these reasons mean that over time, the number of TTI cases decreases among blood donors. However, due to the sensitive nature of blood products, even one case of infection is important and should be detected.

ITS results showed that screening using the new method led to the detection of a higher number of TTIs among the blood donors. If the previous, manual testing method was required to continue, the chances of detecting these cases were extremely low. This change, due to the testing method, was apparent in all TTIs; however, due to the low prevalence of HIV, the changes in the number of the detected cases could not be interpreted.

\section{Conclusion}

This study shows a low prevalence of TTIs (Transfusion Transmitted Infections) among Iranian blood donors. This paper confirms and complements this finding by using the current data for the time period 2003-2017 provided by the Iran Blood Transfusion Organization. The lower prevalence of TTIs compared to other countries can be due to the lower prevalence of TTIs among the general population as well as the voluntary nature of blood donation in Iran. Further statistical analyses using the Interrupted Time Series method provide strong evidences of the effectiveness of modern techniques and procedures for screening the blood donations. This study shows a trend change in the detection of TTIs after replacing manual screening methods by modern automated techniques. The implementation of the new screening method resulted in detecting more cases of TTIs among the blood donors, which increases the overall health of blood products in Iran.

\section{Study Limitation}

In this study, an ELISA was used to diagnose the infection, although it has a specific accuracy, it is currently used to diagnose infections.

\section{Abbreviations}

TTIs, transfusion-transmitted infections; ITS, interrupted time series; IBTO, Iran Blood Transfusion Organization.

\section{Ethics Approval}

In this study, all data were extracted from the national donor registration system. The data were cumulative and there is no individual data from donors available. The current study was approved by the ethical committee at the Kerman University of Medical Sciences, Iran (Ethical Code: IR.KMU.REC.1397.401).

\section{Author Contributions}

All authors made substantial contributions to conception and design, acquisition of data, or analysis and interpretation of data; took part in drafting the article or revising it critically for important intellectual content; gave final approval of the version to be published; and agree to be accountable for all aspects of the work.

\section{Disclosure}

The authors have reported no conflicts of interest in this work.

\section{References}

1. Mohammadali F, Pourfathollah AA. Changes in frequency of HBV, $\mathrm{HCV}, \mathrm{HIV}$ and syphilis infections among blood donors in Tehran province 2005-2011. Arch Iran Med. 2014;17(9):613-620.

2. Flichman DM, Blejer JL, Livellara BI, et al. Prevalence and trends of markers of hepatitis B virus, hepatitis $\mathrm{C}$ virus and human Immunodeficiency virus in Argentine blood donors. BMC Infect Dis. 2014;14(1):218. doi:10.1186/1471-2334-14-218

3. Mohsenizadeh M, Mollaei HR, Ghaziizadeh M. Seroepidemiological study of hepatitis B, C and HIV among blood donors in Kerman. APJCP. 2017;18(12):3267. doi:10.22034/APJCP.2017.18.1.23

4. Kasraian L. National disasters in Iran and blood donation: Bam earthquake experience. Iran Red Crescent Med J. 2010;12(3):316.

5. Bahadorimonfared A, Soori H, Mehrabi Y, et al. Trends of fatal road traffic injuries in Iran (2004-2011). PLoS One. 2013;8(5):e65198. doi:10.1371/journal.pone. 0065198

6. Alavian SM, Ahmadzad-Asl M, Lankarani KB, Shahbabaie MA, Ahmadi AB, Kabir A. Hepatitis C infection in the general population of Iran: a systematic review. Hepat Mon. 2009;9(3).

7. Salehi-Vaziri M, Sadeghi F, Hashiani AA, Fesharaki MG, Alavian SM. Hepatitis B virus infection in the general population of Iran: an updated systematic review and meta-analysis. Hepat Mon. 2016;16 (4). doi:10.5812/hepatmon

8. Saber HR, Tabatabaee SM, Abasian A, et al. Incidence and residual risk of HIV, HBV and HCV infections among blood donors in Tehran. Indian J Hematol Blood Transfus. 2017;33(3):412-416. doi:10.1007/ s12288-016-0732-5

9. Tessema B, Yismaw G, Kassu A, et al. Seroprevalence of HIV, HBV, $\mathrm{HCV}$ and syphilis infections among blood donors at Gondar University Teaching Hospital, Northwest Ethiopia: declining trends over a period of five years. BMC Infect Dis. 2010;10(1):111. doi:10.1186/14712334-10-111 
10. Sajjadi SM, Pourfathollah AA, Mohammadi S, Nouri B, Hassanzadeh R, Rad F. The prevalence and trends of hepatitis B, hepatitis C, and HIV among voluntary blood donors in kohgiluyeh and boyer-ahmad transfusion center, Southwestern Iran. Iran J Public Health. 2018;47(7):944.

11. Bernal JL, Cummins S, Gasparrini A. Interrupted time series regression for the evaluation of public health interventions: a tutorial. Int J Epidemiol. 2017;46(1):348-355. doi:10.1093/ije/dyw098

12. Gómez LA, Penuela O, Higuera F. Prevalence of antibodies against transfusion-transmissible infections (TTI) in blood donors from the Colombian eastern region. Clin Lab. 2014;60(5):869-871. doi:10.7754/clin.lab.2013.130703

13. Gurol E, Saban C, Oral O, Cigdem A, Armagan A. Trends in hepatitis $\mathrm{B}$ and hepatitis $\mathrm{C}$ virus among blood donors over 16 years in Turkey. Eur J Epidemiol. 2006;21(4):299-305. doi:10.1007/s10654-006-0001-2

14. Yadav B, Varma A, Singh P, Kumar R, Bandi P. Seroprevalence of Transfusion-Transmitted Infections (TTIs) in blood donors: a study from Central India. Int J Med Sci Public Health. 2016;5(6):1-5. doi:10.5455/ijmsph.

15. Romanò L, Velati C, Cambiè G, et al. Hepatitis B virus infection among first-time blood donors in Italy: prevalence and correlates between serological patterns and occult infection. Blood Transfus. 2013;11(2):281.

16. Schweitzer A, Horn J, Mikolajczyk RT, Krause G, Ott JJ. Estimations of worldwide prevalence of chronic hepatitis B virus infection: a systematic review of data published between 1965 and 2013. Lancet. 2015;386(10003):1546-1555. doi:10.1016/S0140-6736(15)61412-X
17. Amini Kafi-abad S, Rezvan H, Abolghasemi H, Talebian A Prevalence and trends of human immunodeficiency virus, hepatitis B virus, and hepatitis C virus among blood donors in Iran, 2004 through 2007. Transfusion (Paris). 2009;49(10):2214-2220. doi:10.1111/j.1537-2995.2009.02245.x

18. Mahdaviani F, Saremi S, Maghsoudlu M, Pourfathollah AA Prevalence of blood transmitted viral infections in regular and non-regular donors of Arak Blood Center. Sci JIBTO. 2006;2 (7):343-351.

19. Kasraiyan L, Torab Gahromi SA. Frequency of blood donation for HIV check up at Shiraz Blood Transfusion Organization. JMUMS. 2006;16(51):95-101.

20. Musavi H, Rahimi H, Kooti W, et al. Prevalence of human immunodeficiency virus in Iranian blood donors: a systematic review and meta-analysis. Arch Iran Med. 2018;21(6):260.

21. Taherkhani R, Farshadpour F. Epidemiology of hepatitis $\mathrm{C}$ virus in Iran. World J Gastroenterol. 2015;21(38):10790. doi:10.3748/wjg. v21.i38.10790

22. Cheraghali A. Overview of blood transfusion system of Iran: 2002-2011. Iran J Public Health. 2012;41(8):89.

23. Maghsudlu M, Nasizadeh S, Abolghasemi H, Ahmadyar S. Blood donation and donor recruitment in Iran from 1998 through 2007: ten years' experience. Transfusion (Paris). 2009;49(11):2346-2351. doi:10.1111/trf.2009.49.issue-11

24. Mousavi F, Tavabi A, Golestan B, et al. Knowledge, attitude and practice towards blood donation in Iranian population. Transfus Med. 2011;21(5):308-317. doi:10.1111/j.1365-3148.2011.01080.x
Journal of Blood Medicine

\section{Publish your work in this journal}

The Journal of Blood Medicine is an international, peer-reviewed, open access, online journal publishing laboratory, experimental and clinical aspects of all aspect pertaining to blood based medicine including but not limited to: Transfusion Medicine; Blood collection, Donor issues, Transmittable diseases, and Blood banking logistics; Immunohematology; Artificial and alternative blood based therapeutics; Hematology; Biotechnology/nanotechnology of blood related medicine; Legal aspects of blood medicine; Historical perspectives. The manuscript management system is completely online and includes a very quick and fair peer-review system. Visit http://www.dovepress.com/testimonials.php to read real quotes from published authors. 